\title{
Embryonic Heart
}

National Cancer Institute

\section{Source}

National Cancer Institute. Embryonic Heart. NCI Thesaurus. Code C34153.

The rudimentary heart found in the human embryo that begins development during the third week of gestation, and which is formed by paired endocardial heart tubes derived from splanchnic mesenchyme, which subsequently fuse to form the primitive heart. 